\title{
Cognitive Behavioral Therapy Reduces Benzodiazepine Anxiolytics Use in Japanese Patients with Mood and Anxiety Disorders: A Retrospective Observational Study
}

This article was published in the following Dove Press journal:

Neuropsychiatric Disease and Treatment

\author{
Aiichiro Nakajima' \\ Ayako Kanie' \\ Masaya Ito (iD) \\ Naotsugu Hirabayashi $\mathbb{( \mathbb { D }}^{2}$ \\ Fumi Imamura ${ }^{3}$ \\ Yoshitake Takebayashi ${ }^{1,4}$ \\ Masaru Horikoshi \\ 'National Center for Cognitive Behavior \\ Therapy and Research, National Center \\ of Neurology and Psychiatry, Tokyo, \\ Japan; ${ }^{2}$ Department of Psychiatry, \\ National Center of Neurology and \\ Psychiatry, Tokyo, Japan; ${ }^{3}$ Department of \\ Clinical Psychology, National Center of \\ Neurology and Psychiatry, Tokyo, Japan; \\ ${ }^{4}$ Department of Health Risk \\ Communication, School of Medicine, \\ Fukushima Medical University, Fukushima, \\ Japan
}

Correspondence: Aiichiro Nakajima National Center for Cognitive Behavior Therapy and Research, National Center of Neurology and Psychiatry, 4-I-I, Ogawahigashi, Kodaira, Tokyo I87-855I, Japan

Tel +8I-42-34|-27|I

Fax +8I-42-346-1944

Email aiichiro.nakajima@ncnp.go.jp
Purpose: Benzodiazepines (BZDs) are prescribed to treat psychiatric diseases. However, many guidelines recommend limiting the use of BZDs because of side effects and lack of evidence regarding long-term efficacy. Moreover, reducing BZDs' use is difficult because of dependency and the severity of withdrawal symptoms. The efficacy of cognitive behavioral therapy (CBT) for mood and anxiety disorders has been demonstrated. However, there is scant evidence that CBT has effectively reduced BZDs use, especially in Japan, where the BZDs prescription rate is high. Therefore, we sought to examine the impact of CBT on reducing BZDs use in a Japanese psychiatric setting.

Patients and Methods: Participants were outpatients with mood and anxiety disorders who were prescribed BZD anxiolytics. We retrospectively reviewed changes in BZD anxiolytics prescription dosages during CBT (66 patients; mean number of CBT sessions, 14.6) from our hospital record between April 2015 and September 2017. We checked prescriptions at four time points: at first interview for judging adaptation of CBT (baseline), at the first CBT session, at the last CBT session, and 3 months after the last CBT session.

Results: A total of 13 of 66 patients discontinued BZD anxiolytics during CBT, and 21 of 66 reduced their prescribed dosage by $50 \%$. The association between discontinuation and dosereduction and assessment period was modeled simultaneously using Bayesian hierarchical hurdle model. Results from the modeling showed a significant discontinuation at post-CBT and at 3 months post-CBT session compared to baseline (estimated median odds ratio [OR] post-CBT $=9.79[95 \%$ CI: 4.65-20.45]; OR at 3 months post-CBT $=11.53[95 \% \mathrm{CI}$ : 6.06-22.33]). Moreover, a significant dose reduction was observed post-intervention (estimated median relative risk $=0.845$ [95\% CI: 0.729-0.982]), with a median reduction of $1.7 \mathrm{mg}$ (diazepam conversion) in BZD use.

Conclusion: Our results suggest that CBT possibly aids in reducing and discontinuing BZD anxiolytics use for Japanese patients.

Keywords: psychiatric diseases, depression, acquired dependency, discontinuation

\section{Introduction}

Benzodiazepine (BZD) anxiolytics are commonly used to treat many mental disorders and neurological diseases. In particular, they are prescribed for anxiety symptoms caused by mood and anxiety disorders worldwide. ${ }^{1}$ Previous research has demonstrated the short-term efficacy of BZDs for generalized anxiety 
disorder, panic disorder, and insomnia. ${ }^{2-4}$ However, guidelines do not recommend prescribing BZDs as a firstline treatment for anxiety disorders. Rather, BZDs should only be used for short-term treatment owing to the risk of dependency, memory impairment, and daytime drowsiness. ${ }^{5,6}$ Nevertheless, the use of BZD anxiolytics is often prolonged due to the chronic nature of anxiety, dependency, and severity of withdrawal symptoms. Although present guidelines do not recommend BZD anxiolytics for depressive disorders, BZD anxiolytics have been extensively prescribed for patients with depressive disorders worldwide. ${ }^{7,8}$ Moreover, BZD anxiolytics are considered as avoidance and, as such, are not recommended during exposure therapy as they contribute to disease worsening.

BZD anxiolytics are used frequently in Japan. For instance, BZD anxiolytics consumption, expressed in a defined daily dose (DDD) for statistical purposes (S-DDD), is the second highest (24 S-DDD/1000 inhabitants/day) in Asia after Iran (27 S-DDD/1000 inhabitants/ day). ${ }^{9}$ In addition, Nakagawa et al (2015) have reported that Japanese psychiatrists tend to prescribe BZD anxiolytics for milder mood disorders than do psychiatrists in the United States. ${ }^{10}$ The usual approach for discontinuing the use of long-term BZDs involves a tapered discontinuation; however, it is not particularly effective for achieving longterm discontinuation when the tapered discontinuation is used alone. $^{11-15}$

Cognitive behavioral therapy (CBT) is effective for the treatment of many psychiatric disorders, including anxiety disorders. ${ }^{16}$ CBT is typically conceptualized as a shortterm skill-focused therapy aimed at modifying maladaptive emotional responses by changing patient thoughts, behaviors, or both. However, few studies have investigated whether CBT is helpful in reducing the use of BZD anxiolytics. ${ }^{17,18}$ In Japan, relevant evidence is lacking, as CBT is used less commonly than in Western countries.

Therefore, we conducted a retrospective observational research study in a national psychiatric hospital in Japan. In the hospital, CBT is used to treat psychiatric diseases. Our primary aim was to examine whether CBT would affect the discontinuation or dose-reduction of BZD anxiolytics. We compared the BZD anxiolytics prescription dose before and after the CBT session. Our secondary aim was to examine the prescription of other drugs, antidepressants, hypnotics and, antipsychotics. Moreover, we investigated factors affecting changes in the prescription dose.

\section{Patients and Methods}

\section{Setting and Procedures}

We conducted this research following the principles outlined in the Declaration of Helsinki. Owing to the retrospective nature of the study, the need for informed consent was waived. However, we disclosed details regarding the study on our website, and all participants were offered the opportunity to opt out of the study. This study was approved by the Institutional Review Board at the National Center of Neurology and Psychiatry (NCNP). The NCNP is a leading hospital in the field of psychiatry and neurology in Japan and treats both inpatients and outpatients, including provision of secondary and tertiary treatments. For this study, a retrospective examination of outpatients referred to the CBT department was performed. Outpatients were referred from other departments in our hospital (psychiatry, neurology, and child neurology departments) or other mental hospitals and clinics. In their first interview with psychiatrists in our department, referred patients were assessed regarding whether $\mathrm{CBT}$ was appropriate for them. We provided CBT to most referred patients; however, patients with low motivation to participate in CBT, those with severe intellectual disabilities, or those with strong suicidal ideation were not well suited for CBT and were therefore excluded. If the patient was deemed suitable for CBT, then CBT was scheduled. However, several weeks were usually needed until starting CBT after the first interview.

The present investigation included referred outpatients who were treated between April 2015 and September 2017. We included individuals with mood and anxiety disorders (disorders described in F3 and F4 of the International Statistical Classification of Diseases and Related Health Problems, tenth revision [ICD-10]) who were regularly prescribed BZD anxiolytics and were treated with individual CBT. ${ }^{19}$ We excluded patients who were prescribed BZD anxiolytics only as required because it was difficult to accurately calculate the dosage of BZD anxiolytics per day. We subsequently checked prescriptions at four time points based on hospital records: at first interview for judging adaptation of CBT (baseline), at the first CBT session, at the last CBT session, and 3 months after the last CBT session. Ultimately, we only included patients where there was available information related to prescriptions at the first and last CBT sessions. 


\section{Treatment}

CBT was provided in our department and comprised the following basic components: psychoeducation, selfmonitoring, cognitive restructuring by identifying and challenging emotion-provoking thoughts, systematic exposure to feared situations or stimuli, and practicing problem-solving to cope with anticipated challenges. CBT has a structured format, consisting of weekly sessions for 8-16 weeks. More specifically, exposure and response prevention and cognitive processing therapy were used for patients with obsessive-compulsive disorders and posttraumatic stress disorders, respectively. ${ }^{20,21}$ Patients with more than two diagnoses, such as panic and mood disorders, have largely undergone a unified protocol of the transdiagnostic treatment for emotional disorders. ${ }^{22}$

CBT was implemented by trained psychologists and psychiatrists in our department. Group supervisions helped maintain the quality of the CBT sessions. The group supervisions were provided twice weekly by experienced psychiatrists and psychologists who were trained in the United States.

\section{Data Collection}

To determine the prescribed dose of BZD anxiolytics, we employed a psychotropic dose equivalence table. ${ }^{23}$ To measure drug consumption, DDDs were based on the method of the World Health Organization's (WHO) Collaborative Center for Drug Statistics Methodology, which is used worldwide. However, the WHO warns that DDDs cannot be used as a measure of dose equivalence, as DDD estimations differ among drugs. ${ }^{24}$ Although some psychotropic dose equivalence tables exist, there are racial differences in pharmacodynamics, and some psychotropic drugs are only used in Japan. Therefore, we selected Inada's dose equivalence table, which has been used generally for Japanese clinicians and researchers, for organizing our data. ${ }^{23}$

We collected medication data from hospital records, including data concerning the use of BZD anxiolytics, hypnotics, antidepressants, and antipsychotics. Although some patients with mood and anxiety disorders use mood stabilizers, there are no dose equivalence tables for mood stabilizers; thus, we did not collect data for these drugs. Furthermore, ramelteon and suvorexant, which are not BZD hypnotics, have been available since 2010 and 2014 in Japan, respectively. However, no comparative doubleblind studies with other hypnotics have been reported in
Japan; thus, they are not included in the dose equivalence table, and we did not collect data for these drugs.

We collected the following patient characteristics from medical records: age, sex, education, employment, family living situation, main diagnosis (determined by our trained psychiatrists at the first interview using the Diagnostic and Statistical Manual of Mental Disorders-5, and subsequently checked by other psychiatrists during our regular conferences), disease duration, Beck Depression Inventory (BDI) score at baseline, State-Trait Anxiety Inventory (STAI) score at baseline, CBT protocol, the number of CBT sessions, the duration of CBT sessions, and attitude toward reducing BZD anxiolytics.

\section{Statistical methods}

To estimate the effect of CBT on discontinuation or dosereduction, we compared the prescription-dose at baseline with that after CBT or at follow-up (3 months post-CBT) using a Bayesian hierarchical hurdle regression model. The prescription-dose distribution was expected to be a skewed, non-normal distribution, with values generally concentrated at zero or low dose. Estimates of the gamma and log-normal mixed regression model were compared using information criteria, such as the widely applicable information criterion (WAIC). To compare the baseline and each time point (post-CBT or follow-up), the time point was input into the model as a categorical variable, and estimation was performed using a hierarchical model in which the participants' IDs were set as random effects.

We assumed a different mechanism for discontinuation or dose reduction; generally, discontinuation occurs based on the careful clinical judgment after a certain low-dose period, rather than a gradual linear reduction as a function of time. Dose reduction is expected to decrease gradually. Thus, we assumed a model that simultaneously estimates a binary outcome logistic model, such as whether a threshold is exceeded, as well as a model that explains the linear relationship between outcomes and explanatory variables for samples that exceed the threshold (hurdle). Furthermore, we estimated whether the prescription-dose was zero or not as a withdrawal effect and a change in the prescription amount as a reduction effect. Bayesian hierarchical hurdle regression models were estimated for the prescription dose of BZD anxiolytics, antidepressants, and hypnotics. We included sex and age into the model as covariates.

Markov chain Monte Carlo (MCMC) estimation was applied to the model with default non-informative priors 
using brms package in $\mathrm{R}$ for Bayesian regression models based on Stan package. ${ }^{25}$ We used four chains and performed sampling for each chain 2000 times. We set a 1000-times burn-in duration and obtained 4000 samples in the four chains. Convergence criteria for MCMC were set at $<1.05$ R-hat, which was calculated assuming that a mode of a posterior distribution was a point estimate and that a 95\% confidence interval (CI) was an interval estimate.

We also applied logistic regression to explore factors related to BZD anxiolytics discontinuation during CBT intervention. Factors were possible covariates, including, age, sex, baseline anxiety level (assessed by STAI-S), treatment model (transdiagnostic CBT or diagnosisspecific CBT), number of session, pre-CBT intention for dose-reduction regarding medication, presence of prescription of antidepressant at baseline, presence of prescription of hypnotics at baseline, presence of post-CBT reduction of antidepressant, presence of post-CBT reduction of hypnotics.

\section{Results}

From April 2015 to September 2017, a total of 482 outpatients were referred to our hospital for CBT. There were 160 patients who were diagnosed with mood and anxiety disorders and who were regularly prescribed BZD anxiolytics. Ninety-one of 160 patients were treated with individual CBT, of whom prescription information was available for 66 patients. We included these patients for analysis, but we could not obtain the prescriptions for 3 months post-CBT from 13 patients. The baseline characteristics of participants are shown in Table 1.

The mean time between the first interview and first CBT session (waiting period) and the mean CBT session duration (treatment period) were 66.5 days and 128.5 days, respectively. The doses of BZD anxiolytics, expressed in diazepam conversion, as calculated by the dose equivalence table, were $6.15 \pm 4.5,5.85 \pm 4.5,4.15 \pm 4.4$, and 3.3 $\pm 5.1 \mathrm{mg}$ (median \pm quartile deviation) at the first interview, first CBT session, last CBT session, and at followup, respectively (Table 2). Of 66 patients, 13 discontinued BZD anxiolytics use during $\mathrm{CBT}$, and 21 reduced their BZD anxiolytics use by $50 \%$.

The Bayesian model estimations were appropriate, as all models reached predefined convergence criteria with R-hat in the MCMC estimation. As a result of the model comparison in the WAIC, the hierarchical hurdle lognormal regression model was selected for BZD anxiolytics
Table I Patient Demographics, Symptom Scores at Baseline, and Characteristics

\begin{tabular}{|l|l|}
\hline \multicolumn{2}{|l|}{ N $=66$} \\
\hline Age (years), mean (SD) & $40.8 \pm 12.6$ \\
Women (n, \%) & $37(56 \%)$ \\
More than college degrees (n, \%) & $33(50 \%)$ \\
Employed (n, \%) & $23(35 \%)$ \\
Family living together (n, \%) & $61(92 \%)$ \\
Our hospital's patients (n, \%) & $59(89 \%)$ \\
\hline Primary diagnosis & \\
\hline Mood disorders (n, \%) & $25(38 \%)$ \\
\hline Anxiety disorders (n, \%) & $41(62 \%)$ \\
\hline Disease duration (years), mean (SD) & $9.7 \pm 8.9$ \\
\hline Attitude toward reducing BZD anxiolytics (N = 49) & \\
Positive attitude (n, \%) & $28(42 \%)$ \\
\hline Negative attitude (n, \%) & $21(32 \%)$ \\
\hline BDI score, mean (SD) (N = 48) & $26.2 \pm 12.5$ \\
STAl(state) score, mean (SD) (N = 49) & $56.1 \pm 11.4$ \\
STAl(trait) score, mean (SD) (N = 52) & $62.2 \pm 11.3$ \\
CBT duration (days), mean (SD) & $140.3 \pm 64$ \\
Number of sessions (n), mean (SD) & $14.6 \pm 4.8$ \\
\hline CBT protocols & \\
CBT tailored to the patient (n, \%) & $29(44 \%)$ \\
Exposure and response prevention (n, \%) & $13(20 \%)$ \\
Cognitive processing therapy (n, \%) & $4(6 \%)$ \\
Unified protocol (n, \%) & $20(30 \%)$ \\
\hline
\end{tabular}

Abbreviations: BDI, Beck Depression Inventory; STAI, State-Trait Anxiety Inventory; $\mathrm{CBT}$, cognitive behavioral therapy; SD, standard deviation.

and hypnotics (WAIC were 856.91 for the gamma model and 795.83 for the lognormal model on BZD anxiolytics; WAIC were 375.54 for the gamma model and 349.72 for the lognormal model on hypnotics), and the hierarchical hurdle gamma model was adopted for antidepressants (WAIC were 1777.77 for the gamma model and 1781.45 for the lognormal model). The posterior predictive distribution (PPD) of each model is indicated in Figure 1; the reflected PPD for the selected model seemed to the observed data.

\section{CBT Effects on the Use of BZD Anxiolytics}

Estimation from the hierarchical hurdle lognormal model showed a significant discontinuation of BZD anxiolytics post-CBT and at follow-up compared to baseline (estimated median odds ratio $[\mathrm{OR}]$ after $\mathrm{CBT}=9.79[95 \%$ CI: 4.65-20.45]; OR at 3 months post-CBT $=11.53$ [95\% CI: 6.06-22.33]), but no difference was observed in the 
Table 2 The Doses of Medication Use at the First Interview, Before CBT, After CBT, and 3 Months After CBT (Median \pm Quartile Deviation)

\begin{tabular}{|l|l|l|l|l|}
\hline \multirow{2}{*}{ N = 66 } & \multicolumn{2}{l|}{ Drugs } & AD & HD \\
\cline { 2 - 5 } & BZD Anxiolytics & $50.3 \pm 58.6$ & $0 \pm 2.5$ & AP \\
\hline First Interview (mg/day) & $6.15 \pm 4.5$ & $50.3 \pm 58.6$ & $0 \pm 2.5$ & $0 \pm 42.5$ \\
Before CBT (mg/day) & $5.85 \pm 4.5$ & $50.3 \pm 58.4$ & $0 \pm 2.1$ & $0 \pm 37.5$ \\
After CBT (mg/day) & $4.15 \pm 4.4$ & $83 \pm 56.4$ & $0 \pm 2.5$ & $0 \pm 20.3$ \\
3 months after CBT (mg/day) & $3.3 \pm 5.1$ & & & $0 \pm 21.9$ \\
Discontinuing drug (n, \%) & $13(20 \%)$ & & \\
Reducing drug by 50\% (n, \%) & $21(32 \%)$ & & \\
\hline
\end{tabular}

Notes: As we targeted the prescription of benzodiazepine anxiolytics, some participants were not prescribed other drugs. The number of participants who were prescribed each drug at the first interview are 48 (AD), 27 (HD), and 22 (AP) Table 2.

Abbreviations: BZD anxiolytics, benzodiazepine anxiolytics; $A D$, antidepressant; HD, hypnotic drug; AP, antipsychotic drug.

interval between baseline and before the intervention (estimated median OR $=5.45$ [95\% CI: 0.73-15.67]; Table 3). Moreover, a significant dose reduction was observed post-intervention (estimated median relative risk $=0.845 \quad[95 \%$ CI: $0.729-0.982])$. Although the point estimation for the reduction effect was on a value reflecting the reduction, the credible interval contained zero with high uncertainly.

\section{CBT Effects on the Use of Antidepressants and Hypnotics}

There was no significant effect for discontinuation or dose reduction of antidepressants and hypnotics either during the intervention period or between the period before the intervention up to follow-up.

\section{Factors Associated with BZD Anxiolytics Discontinuation}

We used logistic regression to explore factors associated with BZD anxiolytics discontinuation during CBT intervention. The results indicated that none of the examined factors were significantly associated with BZD anxiolytics discontinuation (Table 4).

\section{Discussion}

Our examination revealed a significant discontinuation of BZD anxiolytics and reduction in their use during and following CBT.

A previous study suggested that a high dosage of BZDs ( $>5 \mathrm{mg} / \mathrm{day})$ is an important predictor of long-term BZDs
BZD anxiolytics

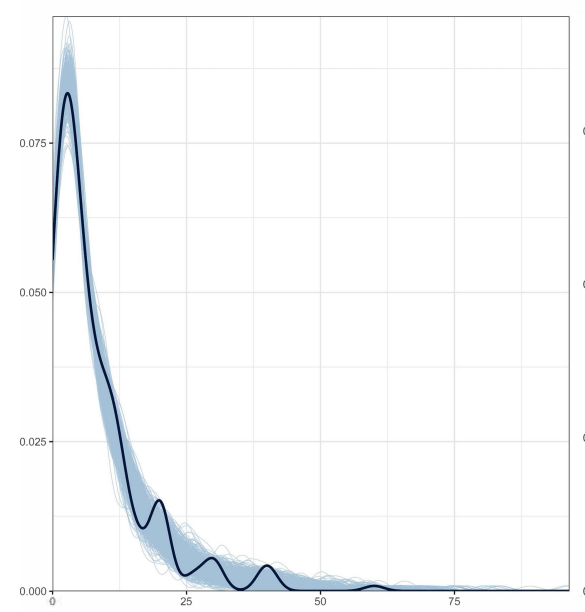

antidepressants

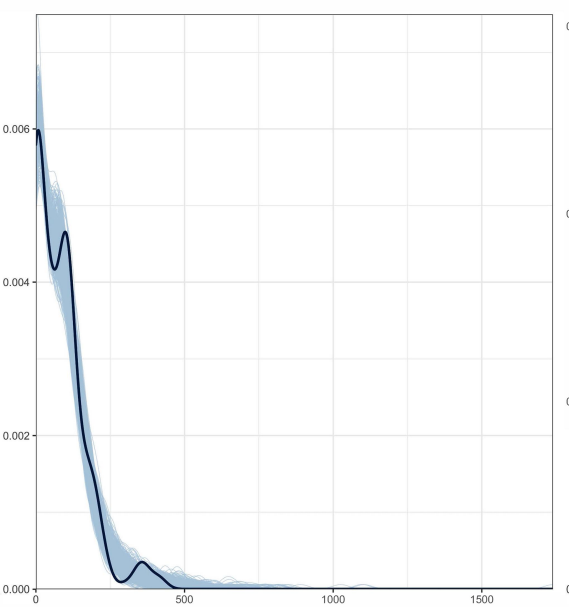

hypnotics

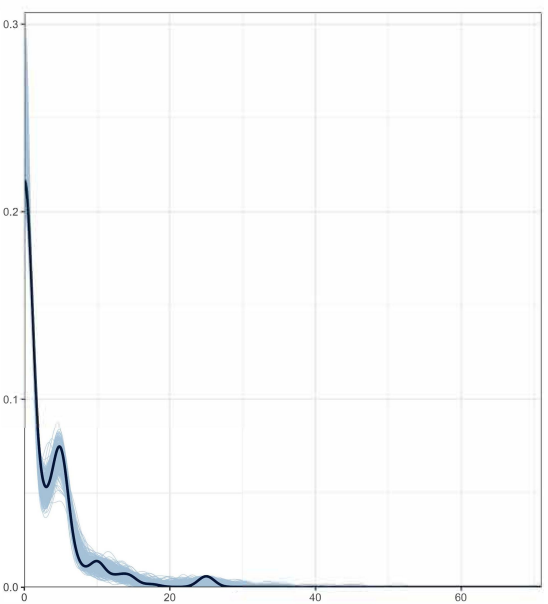

Figure I Post-predictive distribution of the hurdle lognormal (or gamma) model for the prescription dose of BZD anxiolytics, antidepressants, and hypnotics. The x-axis indicates the prescription dose, and the $y$-axis indicates the density of the number of people who are prescribed drugs. The blue range shows post-predictive distribution, and the continuous line shows observed data. This figure shows that observed data of this study are in the posterior predictive distribution for the selected model. Abbreviation: BZD, benzodiazepine. 
Table 3 Estimated Median and Credible Interval from Posterior Distribution Based on Hierarchical Hurdle Lognormal (BZDs, Hypnotics) or Gamma (Antidepressants) Regression Model

\begin{tabular}{|c|l|l|l|l|l|l|}
\hline & \multicolumn{2}{l|}{$\begin{array}{l}\text { Model for } \\
\text { Discontinuation }\end{array}$} & \multicolumn{2}{l|}{ Model for Reduction } \\
\hline & \multicolumn{2}{|l|}{$\begin{array}{l}\text { Odds Ratio [95\% } \\
\text { Credible Interval] }\end{array}$} & \multicolumn{3}{l|}{\begin{tabular}{l}
\multicolumn{2}{l|}{ Relative Risk [95\% } \\
Credible Interval]
\end{tabular}} \\
\hline $\begin{array}{l}\text { BZDs } \\
\text { Before CBT }\end{array}$ & 5.45 & 0.73 & 15.67 & 0.998 & 0.930 & 1.075 \\
After CBT & 9.79 & 4.65 & 20.45 & 0.845 & 0.729 & 0.983 \\
3 months after & 11.53 & 6.06 & 22.33 & 0.888 & 0.773 & 1.013 \\
CBT & & & & & & \\
\hline Antidepressants & & & & & & \\
Before CBT & 0.002 & -1.807 & 1.801 & 1.005 & 0.901 & 1.120 \\
After CBT & 2.171 & 0.486 & 4.311 & 0.993 & 0.832 & 1.178 \\
3 months after CBT & 0.550 & -1.261 & 2.548 & 0.993 & 0.817 & 1.206 \\
\hline Hypnotics & & & & & & \\
Before CBT & 0.541 & -0.884 & 1.932 & 1.060 & 0.961 & 1.165 \\
After CBT & 0.239 & -1.188 & 1.700 & 0.955 & 0.805 & 1.121 \\
3 months after CBT & -0.114 & -1.622 & 1.404 & 1.038 & 0.868 & 1.220 \\
\hline
\end{tabular}

Note: Estimated values were obtained from the model adjusted for age and sex as covariates.

Abbreviations: BZDs, benzodiazepines; CBT, cognitive behavior therapy.

use. ${ }^{18}$ In the present study, patients used higher dosages of BZD anxiolytics $(6.15 \mathrm{mg} /$ day $)$; therefore, it is possible that dose reduction would be difficult in this cohort. Although we expected that hypnotic use would also be reduced during $\mathrm{CBT}$, it remained unchanged. We consider that the low prescription dose of hypnotics at baseline (median, 0) may have affected this result. Moreover, a more specific CBT, such as CBT for insomnia, which is recommended for chronic insomnia, may be needed to reduce hypnotic use. ${ }^{26}$

Our results on the discontinuation of BZD anxiolytics and their use reduction by CBT are consistent with those of previous studies that conducted CBT studies for generalized anxiety disorder and panic disorder. ${ }^{17,18}$ However, the effect size was different between our study and these previous studies. While 13 out of 66 participants (20\%) discontinued BZD anxiolytics in our study, 23 out of 31 participants (74\%) and 9 out of 16 participants (56\%) discontinued BZD anxiolytics in the studies by Gosselin et al and Otto et al, respectively. In these previous studies, participants who wanted to cease their BZD anxiolytics had been recruited. Moreover, the interventions comprised not only CBT but also the slow tapering of BZD anxiolytics. In contrast, in our study, not all participants had a desire to discontinue their medicines, and the decision to reduce BZD anxiolytics was left to the prescribing doctor and participants. These reasons might explain the smaller effect size observed in our study compared to that in the previous studies. However, our findings are useful and indicate that CBT in real-world clinical practice is also effective in reducing the use of BZD anxiolytics and their discontinuation.

A possible explanation for the finding that participants reduce and discontinue BZD anxiolytics during CBT and that this reducing effect remains after the end of CBT sessions is that they can learn how to manage their anxiety symptoms during treatment. Otto et al (2002) indicated that BZD anxiolytics just suppress anxiety symptoms, and if patients reduce them, they feel anxiety again, especially patients with panic disorder. ${ }^{27}$ The authors also

Table 4 Logistic Regression Analysis to Explore Factors Related to Discontinuation for BZDs

\begin{tabular}{|c|c|c|c|c|c|c|}
\hline \multirow[t]{3}{*}{ Predictors } & \multicolumn{3}{|c|}{ Pre-CBT to Post-CBT } & \multicolumn{3}{|c|}{ Pre-CBT to Follow-Up } \\
\hline & \multirow[b]{2}{*}{ OR } & \multicolumn{2}{|l|}{$95 \% \mathrm{Cl}$} & \multirow[b]{2}{*}{ OR } & \multicolumn{2}{|l|}{$95 \% \mathrm{Cl}$} \\
\hline & & Lower Limit & Upper Limit & & Lower Limit & Upper Limit \\
\hline (Intercept) & -3.10 & -10.80 & 3.16 & -0.57 & -8.99 & 7.32 \\
\hline Age & -0.03 & -0.12 & 0.05 & -0.08 & -0.22 & 0.01 \\
\hline Sex & 0.93 & -0.92 & 3.13 & 1.43 & -0.75 & 4.07 \\
\hline Pre State Anxiety (STAI-S) & 0.04 & -0.03 & 0.14 & 0.06 & -0.04 & 0.19 \\
\hline Treatment type & 0.59 & -1.56 & 2.86 & 2.57 & -0.37 & 6.46 \\
\hline Number of CBT sessions & -0.13 & -0.41 & 0.13 & -0.15 & -0.51 & 0.16 \\
\hline Intention to reduce prescription dose & 0.61 & -1.10 & 2.48 & -0.49 & -3.00 & 1.84 \\
\hline Presence of prescription of antidepressants pre-CBT & 0.06 & -1.83 & 2.17 & -1.75 & -4.64 & 0.55 \\
\hline Presence of prescription of hypnotics pre-CBT & -1.00 & -3.68 & 1.12 & -2.64 & -6.26 & 0.00 \\
\hline Post-CBT dose reduction of antidepressants & 0.44 & -2.12 & 2.95 & 0.80 & -2.81 & 4.28 \\
\hline Post-CBT dose reduction of hypnotics & 2.09 & -0.58 & 5.29 & 3.21 & -0.70 & 8.56 \\
\hline
\end{tabular}

Abbreviations: BZDs, benzodiazepines; CBT, cognitive behavior therapy; STAI-S, State-Trait Anxiety Inventory-State; Cl, confidence interval; OR, odds ratio. 
suggested that patients may reduce BZD anxiolytics by managing their anxiety symptoms using core skills acquired by CBT, including informational, cognitive, and exposure skills. The CBT used in our study also included these core skills; therefore, we can assume similar effects.

Dose changes in antidepressants and antipsychotics during CBT have rarely been mentioned in previous studies. During our investigation, the dosage of antidepressants and antipsychotics did not change. Some guidelines mention that antidepressants and antipsychotics not only reduce symptoms of depression and anxiety but also prevent relapse of symptoms. ${ }^{28}$ Therefore, clinicians tend not to reduce or discontinue the dosage of these drugs in the short term. This tendency may affect the unchanged dosage of antidepressants and antipsychotics in our study.

This study has some limitations. First, we were unable to quantify the patients' anxiety and to determine the reasons underlying the changes in BZD anxiolytics dose. Second, we did not include a control group. Thus, the precise factors underlying the reduction and discontinuation of BZD anxiolytics are unclear. Lastly, we gathered data from heterogeneous patients, which has both benefits and limitations. Therefore, we cannot categorically state that a specific CBT effectively reduces the use of BZD anxiolytics or that this is observed for patients with specific diagnoses. Nevertheless, the present results suggest that different patients who are prescribed BZD anxiolytics may be able to reduce or discontinue their medicines following individually tailored CBT. Future studies with a unified parameter and stricter research design are needed to examine the efficacy of CBT in reducing BZD anxiolytics use in Japan. Despite these limitations and taking into account that few studies have examined the relationship between CBT and BZD anxiolytics, we consider that our study has important clinical relevance regarding the role of CBT in reducing BZD anxiolytics use.

\section{Conclusion}

CBT may be used to reduce the dosage of BZD anxiolytics and discontinue their use in patients with mood and anxiety disorders in Japan.

\section{Acknowledgments}

The authors thank the staff of the Department of Clinical Psychology in their hospital, National Center for Cognitive Behavior Therapy and Research, and Translational Medical Center.

\section{Disclosure}

The authors have no financial disclosures or other conflicts of interest to declare.

\section{References}

1. Lader M. Benzodiazepines revisited-will we ever learn? Addiction. 2011;106(12):2086-2109.

2. Martin JL, Sainz-Pardo M, Furukawa TA, Martin-Sanchez E, Seoane T, Galan C. Benzodiazepines in generalized anxiety disorder: heterogeneity of outcomes based on a systematic review and meta-analysis of clinical trials. $J$ Psychopharmacol. 2007;21 (7):774-782. doi:10.1177/0269881107077355

3. van Balkom AJ, Bakker A, Spinhoven P, Blaauw BM, Smeenk S, Ruesink B. A meta-analysis of the treatment of panic disorder with or without agoraphobia: a comparison of psychopharmacological, cognitive-behavioral, and combination treatments. J Nerv Ment Dis. 1997;185(8):510-516. doi:10.1097/00005053-199708000-00006

4. Holbrook A, Crowther R, Lotter A, Endeshaw Y. The role of benzodiazepines in the treatment of insomnia: meta-analysis of benzodiazepine use in the treatment of insomnia. J Am Geriatr Soc. 2001;49 (6):824-826. doi:10.1046/j.1532-5415.2001.49161.x

5. Barker MJ, Greenwood KM, Jackson M, Crowe SF. Cognitive effects of long-term benzodiazepine use: a meta-analysis. CNS Drugs. 2004;18(1):37-48. doi:10.2165/00023210-200418010-00004

6. Canadian Agency for Drugs and Technologies in Health. Short- and Long-Term Use of Benzodiazepines in Patients with Generalized Anxiety Disorder: A Review of Guidelines. Ottawa (ON): Canadian Agency for Drugs and Technologies in Health; 2014.

7. Gelenberg AJ, Freeman MP, Markowitz JC, et al. Practice guideline for the treatment of patients with major depressive disorder, third edition. Am Psychiatry Assoc. 2010;152.

8. Dold M, Bartova L, Mendlewicz J. Clinical correlates of augmentation/combination treatment strategies in major depressive disorder. Acta Psychiatr Scand. 2018;137(5):401-412. doi:10.1111/acps.12870

9. International Narcotics Control Board. Report of the International Narcotics Control Board on the Availability of Internationally Controlled Drugs: Ensuring Adequate Access for Medical and Scientific Purposes. New York: International Narcotics Control Board, United Nations; 2011.

10. Nakagawa A, Williams A, Sado M, et al. Comparison of treatment selections by Japanese and US psychiatrists for major depressive disorder: A case vignette study. Psychiatry Clin Neurosci. 2015;69 (9):553-562. doi:10.1111/pcn.12273

11. Michelini S, Cassano GB, Frare F, Perugi G. Long-term use of benzodiazepines: tolerance, dependence and clinical problems in anxiety and mood disorders. Pharmacopsychiatry. 1996;29 (4):127-134. doi:10.1055/s-2007-979558

12. Salzman C. Benzodiazepine treatment of panic and agoraphobic symptoms: use, dependence, toxicity, abuse. $J$ Psychiatr Res. 1993;27(Suppl 1):97-110. doi:10.1016/0022-3956(93)90021-S

13. Mellman TA, Uhde TW. Withdrawal syndrome with gradual tapering of alprazolam. Am J Psychiatry. 1986;143(11):1464-1466.

14. Pecknold JC, Swinson RP, Kuch K, Lewis CP. Alprazolam in panic disorder and agoraphobia: results from a multicenter trial. III. Discontinuation effects. Arch Gen Psychiatry. 1988;45(5):429-436. doi:10.1001/archpsyc.1988.01800290043006

15. Schweizer E, Rickels K, Case WG, Greenblatt DJ. Carbamazepine treatment in patients discontinuing long-term benzodiazepine therapy. Effects on withdrawal severity and outcome. Arch Gen Psychiatry. 1991;48(5):448-452. doi:10.1001/archpsyc.1991.01810 290060012

16. Butler AC, Chapman JE, Forman EM, Beck AT. The empirical status of cognitive-behavioral therapy: a review of meta-analyses. Clin Psychol Rev. 2006;26(1):17-31. doi:10.1016/j.cpr.2005.07.003 
17. Gosselin P, Ladouceur R, Morin CM, Dugas MJ, Baillargeon L. Benzodiazepine discontinuation among adults with GAD: a randomized trial of cognitive-behavioral therapy. $J$ Consult Clin Psychol. 2006;74(5):908-919. doi:10.1037/0022-006X.74.5.908

18. Otto MW, McHugh RK, Simon NM, Farach FJ, Worthington JJ, Pollack MH. Efficacy of CBT for benzodiazepine discontinuation in patients with panic disorder: further evaluation. Behav Res Ther. 2010;48(8):720-727. doi:10.1016/j.brat.2010.04.002

19. World Health Organization. The ICD-10 Classification of Mental and Behavioural Disorders: Clinical Descriptions and Diagnostic Guidelines. Geneva: World Health Organization; 1992.

20. McKay D, Sookman D, Neziroglu F, et al. Accreditation Task Force of The Canadian Institute for Obsessive Compulsive Disorders. Efficacy of cognitive-behavioral therapy for obsessive-compulsive disorder. Psychiatry Res. 2015;225(3):236-246. doi:10.1016/j. psychres.2014.11.058

21. Resick PA, Monson CM, Chard KM. Cognitive Processing Therapy for PTSD: A Comprehensive Manual. The Guildford Press; 2016.

22. Barlow DH, Farchione TJ, Bullis JR, et al. The unified protocol for transdiagnostic treatment of emotional disorders compared with diagnosis-specific protocols for anxiety disorders: a randomized clinical trial. JAMA Psychiatry. 2017;74(9):875-884. doi:10.1001/ jamapsychiatry.2017.2164
23. Inada $\mathrm{T}$, Inagaki A. Psychotropic dose equivalence in Japan. Psychiatry Clin Neurosci. 2015;69(8):440-447. doi:10.1111/ pcn. 12275

24. Leucht S, Samara M, Heres S, Davis JM. Dose equivalents for antipsychotic drugs: the DDD method. Schizophr Bull. 2016;42 (Suppl 1):S90-4. doi:10.1093/schbul/sbv167

25. Paul-Christian B. brms: an R package for bayesian multilevel models using stan. J Stat Softw. 2017;80(1):1-28.

26. Qaseem A, Kansagara D, Forciea MA, Cooke M, Denberg TD. Management of chronic insomnia disorder in adults; a clinical practice guideline from the American college of physicians. Ann Intern Med. 2016;165:125-133. doi:10.7326/M15-2175

27. Otto MW, Hong JJ, Safren SA. Benzodiazepine discontinuation difficulties in panic disorder conceptual model and outcome for cognitive-behavior therapy. Curr Pharm Des. 2002;8(1):75-80. doi:10.2174/1381612023396726

28. Katzman MA, Bleau P, Blier P, Chokka P, Kjernisted K, Van Ameringen M. Canadian clinical practice guidelines for the management of anxiety, posttraumatic stress and obsessive-compulsive disorders. BMC Psychiatry. 2014;14:S1. doi:10.1186/1471-244X-14S1-S1

\section{Publish your work in this journal}

Neuropsychiatric Disease and Treatment is an international, peerreviewed journal of clinical therapeutics and pharmacology focusing on concise rapid reporting of clinical or pre-clinical studies on a range of neuropsychiatric and neurological disorders. This journal is indexed on PubMed Central, the 'PsycINFO' database and CAS, and is the official journal of The International Neuropsychiatric Association (INA). The manuscript management system is completely online and includes a very quick and fair peer-review system, which is all easy to use. Visit http://www.dovepress.com/testimonials.php to read real quotes from published authors. 